\title{
Outcomes of 27 Non-Small Cell Lung Cancer Patients Receiving Resections of Paired Primary Pulmonary and Brain Metastatic Tumours: Is EGFR Negative Mutation of Brain Metastasis Bring More Benefit?
}

\section{Shuonan Xu}

Air Force Medical University 2nd Affiliated Hospital: Air Force Medical University Tangdu Hospital Jianfei Zhu

Air Force Medical University 2nd Affiliated Hospital: Air Force Medical University Tangdu Hospital Daixing Zhong

Air Force Medical University 2nd Affiliated Hospital: Air Force Medical University Tangdu Hospital Yanmin Xia

Air Force Medical University 2nd Affiliated Hospital: Air Force Medical University Tangdu Hospital Yingsheng Wen

Sun Yat-sen University Cancer Center

Lanjun Zhang

Sun Yat-sen University Cancer Center

Tang Jiang ( $\sim$ jiangtaotd@163.com )

Air Force Medical University 2nd Affiliated Hospital: Air Force Medical University Tangdu Hospital https://orcid.org/0000-0002-0578-9157

\section{Research}

Keywords: epidermal growth factor receptor, gene mutation, Non-small cell lung cancer, brain metastases, heterogeneity

Posted Date: August 17th, 2021

DOl: https://doi.org/10.21203/rs.3.rs-795208/v1

License: (c) (1) This work is licensed under a Creative Commons Attribution 4.0 International License. Read Full License 


\section{Abstract}

Background: To analyze the heterogeneity and clinical outcomes of epidermal growth factor receptor (EGFR) gene mutation in primary tumour and corresponding brain metastasis(BM) in non-small cell lung cancer (NSCLC).

Methods: Primary pulmonary tumours and paired metastatic brain tumours were surgically removed from twenty-seven NSCLC patients from July 1999 to November 2013 in our hospital. All brain lesions were confirmed as metastases stemming from NSCLC by pathological examination. EGFR gene (exons 18-21) mutant status was detected in matched tumour by using amplification refraction mutation system (ARMS). If inconsistency was detected, the paired tumour was evaluated again. The McNemar test was performed to compare the consistency of the paired tumour, and the Kappa test was used to quantify the agreement of both methods.Progression free survival(PFS) and overall survival(OS) were exhibited by the Kaplan-Meier.

Results: In this study, of the 27 patients, nine (33.3\%) cases were found to have EGFR mutations in BMs, and ten $(37.0 \%)$ patients were detected positive EGFR status in primary lung tumour tissue. The rate of consistency of the matched tumour was $24 / 27$ (88.9\%, $\mathrm{P} \leq 0.001)$, and the Kappa coefficient was 0.757. Among the three cases presenting EGFR mutational heterogeneity, two patients harbored EGFR mutation in primary tumors but was negative in BMs, meanwhile, the other patient had the opposite pattern. Comparing to patients with consistent EGFR mutations(both mutant or wild),patients with inconsistent EGFR mutations tended to have better outcomes, including PFS(37.2months vs 25.0 months vs 16.5 months, $P=0.159)$ and $O S(53.6$ months vs 27.5 months vs 26.8 months, $P=0.380)$, further analysis showed that two patients whose EGFR mutant-type primary tumor progressing to wild-type cerebral metastastic tumor might have longer overall survival(53.6months,37.8months) than one patient harboring reverse mutant difference(EGFR wild-type primary tumor progressing to mutant-type brain metastastic tumor) (16.7months). Also we found that patients with wild type in brain metastatic tumour had longer overall survival (OS)(mOS, 36.3 months vs 29.1 months, $P=0.944)$.

Conclusions: EGFR mutation status in NSCLC patients between primary lung tumour and paired BM was heterogeneous, patients harbored wild type EGFR mutation in BM might have better outcomes, especially for positive status transferred to wild.

\section{Introduction}

Brain metastases (BMs) are the leading cause of death in non-small cell lung cancer (NSCLC) patients, and approximately $30 \%$ of patients experience BM during their disease progression[1]. Therapeutic strategies for BM include surgery, whole-brain radiotherapy (WBRT), stereotactic radiosurgery (SRS), chemotherapy, or a combination of these treatments. Although NSCLC patients with BMs have many options for treating this disease, the median overall survival is only approximately 6-8 months[2-4]. Gefitinib, icotinib,and erlotinib, the epidermal growth factor receptor (EGFR) tyrosine kinase inhibitors 
(TKIs), have shown great efficacy in brain metastases in NSCLC because some data indicate that TKIs are able to cross the blood-brain barrier (BBB)[5-7]. Osimertinib, a third generation TKI, is widely used in the treatment of patients harbouring EGFR mutations. Compared with standard TKIs, osimertinib had superior efficacy in intracranial disease control (15.2 months vs 9.6 months, P『0.001)[8]. EGFR gene mutation status has been recognized as the best predictor of the efficacy of TKIs, and some studies have reported that TKIs had obviously different efficiency in different patients, even in one patient's different lesions, and the specific mechanism that drives this was unclear. A T790 mutation, C-MET amplification, and small cell transformation are believed to be the some of the causes of TKI drug resistance and treatment failure[9-11]. Recently, EGFR mutational heterogeneity has been payed more attention as a potential reason for treatment failure[12]. This phenomenon has been reported intratumourally[13], between primary lung lesions and metastatic lymph nodes[14], between primary lung lesions and peripheral circulating tumour cells and in other metastatic lesions[15-17]. Due to the difficulty of sampling brain tumour tissue, there are few studies on the EGFR mutational heterogeneity of brain metastatic tumour and primary lung cancer. Matsumoto et al[18] analyzed the incidence of EGFR mutations in brain metastases of lung adenocarcinoma, and the frequency was $63 \%(12 / 19)$; in the six patients that harbored EGFR mutations, the corresponding primary lung tumour were observed to be of the same mutational type, and no discordant mutation patterns were found in that study. Noticeably,EGFR mutant patients had higher risk developed BM than those with EGFR wild-type.For synchronous BM patients,EGFR positive in primary lung tumour might predict that patients have better survival than wild-type patients,the similar result did not find in metachronous patients[19]. It seem that EGFR mutation involved the course of BM and the change of the EGFR status influenced the outcomes of patients with BMs. Our previous study demonstrated that TKIs combined with conventional therapy might be beneficial for survival of patients with BM; interestingly, TKIs had significantly different efficacy in intracranial lesions and extracranial lesions[20]. In present study, 27 patients underwent brain metastatic tumour resection. The purpose of this study was to find the heterogeneity of EGFR mutations in primary tumour and corresponding brain metastatic tumour,and analyze the relationship between the discordance and the outcomes.

\section{Materials And Methods Patients selection}

Twenty-seven primary NSCLC patients with BMs who underwent treatment at he Second Affiliated Hospital of Air Force Medical University between July 1999 and November 2013 were eligible for inclusion in this study. The cases selected for this study fulfilled the following criteria: (1) histologically or cytologically confirmed primary NSCLC and no other types of tumours in the past; (2) brain metastasis detected by cranial CT, cranial MRI, or both; (3) entire surgical resection for brain metastases; (4) the pathological type of metastatic tumour matched with the primary lung tumour; (5) sufficient primary tissues sampled for micro-dissection and molecular analysis; (6) patients had not received EGFR-TKI 
therapy before the retrieval of tumour tissues; and (7) adequate clinical information and available followup data.

EGFR mutation detection by an ARMS method All of the tissues tested were formalin-fixed paraffinembedded (FFPE) tissues, including the 27 pairs of lung primary tumours and brain metastatic tumours. Tumour cells should be evaluated and presented at more than $1 \%$ before detection. EGFR gene mutations, including 29 common types, were detected using an amplification mutation refractory system (ARMS). All experiments were performed in strict accordance with the kit instructions (Amoy Diagnostics, Xiamen, China). If inconsistency was found, the paired tumours from the different lesions were measured again(Fig. 1). The details of the ARMS method were described in our previous study(20). Statistical analysis Patients were subgrouped by their clinical characteristics. The McNemar test was performed to compare EGFR mutation status between lung primary tumours and metastatic brain tumours, and the Kappa test was performed to quantify the agreement of both methods. EGFR mutation status in lung primary tissue was considered as the point of comparison. Survival analysis was evaluated by the Kaplan-Meier test, and a log-rank test was performed. For variables that were significant in the univariate analysis, multivariate analysis was performed via the Cox regression model. P-value less than 0.05 was considered statistically significant.

\section{Result}

\section{Patient Characteristics}

A total of 27 NSCLC patients with BMs were enrolled in this study, including 22 males and 5 females. The median age of patients at diagnosis of brain metastasis was 55 years (with a range of 29-73 years). Of the 27 patients, fifteen were smokers, and twelve were non-smokers. Pathologic type was determined according to WHO criteria, and 23 patients had adenocarcinoma, 3 had squamous cell carcinoma, and one had sarcomatoid carcinoma. The twenty-seven analyzed metastases were synchronous in 15 cases and metachronous in 12 cases. The median time from the discovery of the primary tumour to brain metastasis was 25.0 months, the longest time for one case was 49.7 month. The other clinical-pathologic characteristics of the 27 patients are listed in Table 1. 
Table 1

Characteristics of NSCLC patients with brain metastases.

\begin{tabular}{|c|c|c|}
\hline Characteristics & $\mathbf{N}$ & $\%$ \\
\hline Age, median (range) & 50 & $29-73$ \\
\hline \multicolumn{3}{|l|}{ Gender } \\
\hline Male & 22 & $81.5 \%$ \\
\hline Female & 5 & $18.5 \%$ \\
\hline \multicolumn{3}{|l|}{ Smoking status } \\
\hline Non-smoker & 12 & $44.4 \%$ \\
\hline Smoker & 15 & $55.6 \%$ \\
\hline \multicolumn{3}{|l|}{ Histology } \\
\hline$A D C^{1}$ & 23 & $85.2 \%$ \\
\hline Non $-A D C^{2}$ & 4 & $14.8 \%$ \\
\hline \multicolumn{3}{|c|}{ AJCC disease stage of primary lung cancer } \\
\hline Stage I & 4 & $7.4 \%$ \\
\hline Stage II & 4 & $14.8 \%$ \\
\hline Stage III & 4 & $14.8 \%$ \\
\hline Stage IV (operable brain metastases) & 15 & $63.0 \%$ \\
\hline \multicolumn{3}{|l|}{ Metastatic type } \\
\hline Synchronous $^{3}$ & 15 & $55.6 \%$ \\
\hline Metachronous $^{4}$ & 12 & $44.4 \%$ \\
\hline \multicolumn{3}{|c|}{$\begin{array}{l}{ }^{1} \mathrm{ADC} \text { :adenocarcinoma; }{ }^{2} \text { Non }-\mathrm{ADC} \text { : non-adenocarcinoma; }{ }^{3} \text { Synchronous:brain metastases } \\
\text { diagnosed with primary lung cancer; }{ }^{4} \text { Metachronous:brain metastases diagnosed during the disease } \\
\text { courses. }\end{array}$} \\
\hline
\end{tabular}

EGFR mutation status between primary lung tumour and corresponding brain metastatic tumour

The EGFR mutation status of the primary tumour and the corresponding metastatic brain tumour is shown in Table 2. EGFR mutation were detected in 37.0\% (10/27) of the primary tumour, and the majority of EGFR mutations were deletions in exon 19 ( 6 of 27, 22.2\%), L858R mutations in exon 21 (3 of 27, $11.1 \%$ ) and G719X mutations in exon 18 (1 of 27, 3.7\%). Nine cases with EGFR mutation in brain metastases included 6 cases of deletion in exon 19, 2 cases of L858R mutation in exon 21 and 1 case of an exon 18 G719X mutation. 
Table 2

Discordant EGFR mutations between primary lung tumor and corresponding brain metastatic tumor.

\begin{tabular}{|c|c|c|c|c|c|c|}
\hline $\begin{array}{l}\text { Case } \\
\text { no. }\end{array}$ & Gender & Age & $\begin{array}{l}\text { Smoking } \\
\text { status }\end{array}$ & Pathology & $\begin{array}{l}\text { EGFR mutation in } \\
\text { primary lung tumour }\end{array}$ & $\begin{array}{l}\text { EGFR mutation in brain } \\
\text { metastatic tumour }\end{array}$ \\
\hline NO.1 & $M$ & 69 & $\begin{array}{l}\text { Non- } \\
\text { smoker }\end{array}$ & $A D C^{1}$ & E746-A750 del & Wild \\
\hline NO.2 & $M$ & 53 & Smoker & $\mathrm{scc}^{2}$ & Wild & Wild \\
\hline NO.3 & M & 70 & Smoker & $A D C$ & Wild & Wild \\
\hline NO.4 & $M$ & 73 & Smoker & $A D C$ & Wild & Wild \\
\hline NO.5 & $F$ & 51 & $\begin{array}{l}\text { Non- } \\
\text { smoker }\end{array}$ & $A D C$ & E746-A750 del & E746-A750 del \\
\hline NO.6 & $M$ & 61 & Smoker & $A D C$ & Wild & Wild \\
\hline NO.7 & $M$ & 58 & Smoker & SCC & L858R & Wild \\
\hline NO.8 & $F$ & 29 & $\begin{array}{l}\text { Non- } \\
\text { smoker }\end{array}$ & $A D C$ & E746-A750 del & E746-A750 del \\
\hline NO.9 & $M$ & 46 & Smoker & $A D C$ & L858R & L858R \\
\hline N0.10 & $M$ & 63 & $\begin{array}{l}\text { Non- } \\
\text { smoker }\end{array}$ & $A D C$ & Wild & E746-A750 del \\
\hline N0.11 & $M$ & 44 & $\begin{array}{l}\text { Non- } \\
\text { smoker }\end{array}$ & $A D C$ & E746-A750 del & E746-A750 del \\
\hline N0.12 & M & 52 & Smoker & $s c^{3}$ & G719X & G719X \\
\hline N0.13 & M & 58 & Smoker & $A D C$ & Wild & Wild \\
\hline N0.14 & $F$ & 53 & Smoker & $A D C$ & E746-A750 del & E746-A750 del \\
\hline N0.15 & $M$ & 40 & Smoker & $A D C$ & Wild & Wild \\
\hline N0.16 & $M$ & 73 & Smoker & $A D C$ & E746-A750 del & E746-A750 del \\
\hline NO.17 & $F$ & 55 & $\begin{array}{l}\text { Non- } \\
\text { smoker }\end{array}$ & $A D C$ & Wild & Wild \\
\hline N0.18 & $M$ & 57 & Smoker & $A D C$ & Wild & Wild \\
\hline N0.19 & $M$ & 53 & Smoker & $A D C$ & Wild & Wild \\
\hline NO.20 & $F$ & 54 & $\begin{array}{l}\text { Non- } \\
\text { smoker }\end{array}$ & $A D C$ & Wild & Wild \\
\hline N0.21 & M & 55 & Smoker & $A D C$ & Wild & Wild \\
\hline
\end{tabular}

${ }^{1} \mathrm{ADC}$ :adenocarcinoma; ${ }^{2} \mathrm{SCC}$ :squamous cell carcinoma; ${ }^{3}$.sarcomatoid carcinoma 


\begin{tabular}{|c|c|c|c|c|c|c|}
\hline $\begin{array}{l}\text { Case } \\
\text { no. }\end{array}$ & Gender & Age & $\begin{array}{l}\text { Smoking } \\
\text { status }\end{array}$ & Pathology & $\begin{array}{l}\text { EGFR mutation in } \\
\text { primary lung tumour }\end{array}$ & $\begin{array}{l}\text { EGFR mutation in brain } \\
\text { metastatic tumour }\end{array}$ \\
\hline NO.22 & $\mathrm{M}$ & 72 & Smoker & $A D C$ & Wild & Wild \\
\hline NO.23 & M & 60 & $\begin{array}{l}\text { Non- } \\
\text { smoker }\end{array}$ & SCC & Wild & Wild \\
\hline NO.24 & $M$ & 66 & Smoker & $A D C$ & Wild & Wild \\
\hline No.25 & $M$ & 61 & $\begin{array}{l}\text { Non- } \\
\text { smoker }\end{array}$ & $A D C$ & Wild & Wild \\
\hline N0.26 & $M$ & 51 & $\begin{array}{l}\text { Non- } \\
\text { smoker }\end{array}$ & $A D C$ & Wild & Wild \\
\hline N0.27 & $\mathrm{M}$ & 49 & $\begin{array}{l}\text { Non- } \\
\text { smoker }\end{array}$ & $A D C$ & L858R & L858R \\
\hline
\end{tabular}

\section{The relationships between EGFR mutation status and patients' clinic-pathological factors.}

Out of the twenty-seven cases of lung primary tumours that were analyzed, EGFR mutations were detected in 10 of the 27 cases (37.0\%). The subgroup analysis showed that EGFR gene mutations occurred more frequently in females $(60.0 \%$ vs $31.8 \%, \mathrm{P}=0.326)$ and non-smokers $(50.0 \%$ vs $26.7 \%, \mathrm{P}=$ $0.257)$. Interestingly, the EGFR mutation rate was $34.8 \%(8 / 23)$ in adenocarcinoma, which was lower than that of non-adenocarcinoma, which was $50 \%(2 / 4, P=0.613)$.

Of the 27 cases of paired brain metastatic tumours, EGFR mutations were detected in the brain metastasis tumour tissue of 9 patients, giving a mutation rate of $33.3 \%$. The subgroup analysis found more EGFR mutations in the brain metastases of female NSCLC patients (3/5) compared with those of male patients $(6 / 22)(60.0 \%$ vs $27.3 \%, P=0.295)$. The smoking mutation rate $(3 / 15)$ was lower than the non-smoker mutation rate $(6 / 12,20 \%$ vs $50.0 \%, P=0.127)$. The adenocarcinoma mutation rate was $34.8 \%(8 / 23)$, which was higher than that of the non-adenocarcinoma mutation rate of $25 \%(1 / 4, P=$ 0.636). EGFR gene mutations were high in young patients $(8 / 21,38.1 \%$ vs $1 / 6,16.7 \%, P=0.628)$. The study also found that the rate of EGFR mutation in patients with newly diagnosed brain metastases was $6 / 15(40.0 \%)$, which was higher than that of patients that did not initially present with brain metastases $(3 / 12,25 \%, P=0.683)$.

The heterogeneity of EGFR mutation status in primary lung tumours and corresponding brain metastases.

The EGFR mutation status in the primary lung tumour and the corresponding brain metastatic sites of each patient was not always identical (Table 3). EGFR mutations were discordant in $11.1 \%$ of paired lesions ( 3 of 27 cases). Two cases (7.4\%) showed EGFR mutations in the primary tumours but not in the metastatic brain tumour. One case $(3.7 \%)$ showed EGFR mutations in the brain metastatic tumour but not 
in the primary tumour. The same results were obtained by a second analysis for different parts of the tumour (Fig. 1). According to the results of the analysis by the McNemar test, the EGFR gene mutations in primary tumour tissue and brain metastases were significantly different $(P \leq 0.001)$. The kappa coefficient was 0.757. (Table 4). 
Table 3

The relationships between the EGFR mutation status and clinic-pathological factors.

\begin{tabular}{|c|c|c|c|c|}
\hline Variable & $\begin{array}{l}\text { EGFR mutation in primary } \\
\text { lung tumor }\end{array}$ & $p$ & $\begin{array}{l}\text { EGFR mutation in brain } \\
\text { metastases tumor }\end{array}$ & $p$ \\
\hline Gender & & 0.326 & & 0.295 \\
\hline Male & 7 & & 6 & \\
\hline Female & 3 & & 3 & \\
\hline Age (years) & & 1.000 & & 0.628 \\
\hline$\leq 65$ & 8 & & 8 & \\
\hline$\bigotimes 65$ & 2 & & 1 & \\
\hline Smoking status & & 0.257 & & 0.127 \\
\hline Non-smoker & 6 & & 6 & \\
\hline Smoker & 4 & & 3 & \\
\hline Histology & & 0.613 & & 1.000 \\
\hline $\mathrm{ADC}^{1}$ & 8 & & 8 & \\
\hline Non-ADC ${ }^{2}$ & 2 & & 1 & \\
\hline Diameter of BM & & 1.000 & & 0.695 \\
\hline 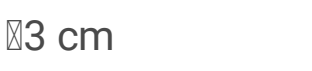 & 5 & & 5 & \\
\hline$\geq 3 \mathrm{~cm}$ & 5 & & 4 & \\
\hline $\begin{array}{l}\text { Intracranial } \\
\text { symptom }\end{array}$ & & 0.683 & & 0.423 \\
\hline Yes & 4 & & 5 & \\
\hline No & 6 & & 4 & \\
\hline Metastatic type & & 1.000 & & 0.683 \\
\hline Synchronous 3 & 6 & & 6 & \\
\hline Metachronous ${ }^{4}$ & 4 & & 3 & \\
\hline \multicolumn{5}{|c|}{$\begin{array}{l}{ }^{1} \mathrm{ADC} \text { :adenocarcinoma; }{ }^{2} \mathrm{Non}-\mathrm{ADC} \text { : non-adenocarcinoma; }{ }^{3} \text { Synchronous:brain metastases } \\
\text { diagnosed with primary lung cancer; }{ }^{4} \text { Metachronous:brain metastases diagnosed during the disease } \\
\text { courses. }\end{array}$} \\
\hline
\end{tabular}


Table 4

Comparison of EGFR genotypes between primary lung tumors and corresponding brain metastases tumors in NSCLC with brain metastases.

\begin{tabular}{|llll|}
\hline Primary lung tumors & & Brain metastases tumors & In total \\
\hline & Mutant & Wild & \\
\hline Mutation & 8 & 2 & 10 \\
\hline Wild & 1 & 16 & 17 \\
\hline In total & 9 & 18 & 27 \\
\hline Kappa value:0.757, $P \leq 0.001$ & & \\
\hline
\end{tabular}

\section{Survival analysis according to clinical-pathological factors in NSCLC patients.}

Univariate analysis showed that the following variables correlated with patient survival: histology and metastatic type(Table 5). EGFR mutation status in primary lung tumour was analyzed,patients harbored EGFR mutant seemed to have longer PFS (mPFS,27.8months vs 17.4 months,P $=0.156$ ) and OS (mPFS,34.9months vs 27.6 months,P $=0.606$ )(Fig. 2).For matched brain metastatic tumour, patients with negative mutations had a longer OS of 34.9 months compared with 27.6 months in EGFR positive patients $(P=0.944)$,although this kind patients had unfavorable PFS(mPFS,20.6months vs 26.4 months, $P$ $=0.325)$ (Fig. 3) . 
Table 5

Kaplan-Meier survival analysis (log-rank test) according to clinical-pathological factors in NSCLC patients with BM.

\begin{tabular}{|c|c|c|c|c|c|c|c|}
\hline Variable & $N$ & PFS(months) & $95 \% \mathrm{Cl}$ & $P$ & OS(months) & $95 \% \mathrm{Cl}$ & $P$ \\
\hline \multirow{2}{*}{$\begin{array}{l}\text { In total } \\
\text { Gender }\end{array}$} & 27 & 23.0 & $\begin{array}{l}16.6- \\
29.4\end{array}$ & & 32.9 & $\begin{array}{l}23.9- \\
41.8\end{array}$ & \\
\hline & & & & 0.275 & & & 0.252 \\
\hline Male & 22 & 21.1 & $\begin{array}{l}14.6- \\
27.5\end{array}$ & & 31.8 & $\begin{array}{l}21.4- \\
42.4\end{array}$ & \\
\hline Female & 5 & 28.8 & $\begin{array}{l}14.5- \\
43.2\end{array}$ & & 34.9 & $\begin{array}{l}22.7- \\
47.1\end{array}$ & \\
\hline Age & & & & 0.081 & & & 0.610 \\
\hline$\leq 65$ & 21 & 25.3 & $\begin{array}{l}18.0- \\
32.6\end{array}$ & & 30.3 & $\begin{array}{l}23.2- \\
37.4\end{array}$ & \\
\hline$\otimes 65$ & 6 & 16.0 & $\begin{array}{l}2.8- \\
29.2\end{array}$ & & 31.6 & $\begin{array}{l}11.3- \\
52.0\end{array}$ & \\
\hline Smoking status & & & & 0.107 & & & 0.208 \\
\hline Non-smoker & 12 & 27.7 & $\begin{array}{l}18.2- \\
37.0\end{array}$ & & 38.2 & $\begin{array}{l}25.2- \\
51.2\end{array}$ & \\
\hline Smoker & 15 & 19.3 & $\begin{array}{l}11.5- \\
27.0\end{array}$ & & 24.2 & $\begin{array}{l}16.7- \\
31.6\end{array}$ & \\
\hline Histology & & & & 0.042 & & & 0.001 \\
\hline$A C^{1}$ & 23 & 13.3 & $\begin{array}{l}17.8- \\
31.2\end{array}$ & & 35.7 & $\begin{array}{l}26.3- \\
45.1\end{array}$ & \\
\hline $\mathrm{NAC}^{2}$ & 4 & 24.5 & $0-27.3$ & & 14.1 & $\begin{array}{l}0.6- \\
27.6\end{array}$ & \\
\hline $\begin{array}{l}\text { EGFR mutation in } \\
\text { primary lung tumour }\end{array}$ & & & & 0.156 & & & 0.606 \\
\hline Wild & 17 & 17.4 & $\begin{array}{l}11.1- \\
23.7\end{array}$ & & 27.6 & $\begin{array}{l}19.5- \\
35.7\end{array}$ & \\
\hline Mutant & 10 & 27.8 & $\begin{array}{l}17.8- \\
37.8\end{array}$ & & 34.9 & $\begin{array}{l}21.5- \\
48.3\end{array}$ & \\
\hline
\end{tabular}

\footnotetext{
${ }^{1} \mathrm{ADC}$ :adenocarcinoma; ${ }^{2}$ Non -ADC: non-adenocarcinoma; ${ }^{3}$ Synchronous:brain metastases diagnosed with primary lung cancer; ${ }^{4}$ Metachronous:brain metastases diagnosed during the disease courses; ${ }^{5}$ EGFR-TKIs:first generation of tyrosine kinase inhibitors (TKIs): Gefitinib and Erlotinib; ${ }^{6}$ systemic chemotherapy plus local treatment.
} 


\begin{tabular}{|c|c|c|c|c|c|c|c|}
\hline Variable & $\mathbf{N}$ & PFS(months) & $95 \% \mathrm{Cl}$ & $P$ & OS(months) & $95 \% \mathrm{Cl}$ & $P$ \\
\hline $\begin{array}{l}\text { EGFR mutation in } \\
\text { brain metastatic } \\
\text { tumour }\end{array}$ & & & & 0.325 & & & 0.944 \\
\hline Wild & 18 & 20.6 & $\begin{array}{l}13.4- \\
27.8\end{array}$ & & 36.3 & $\begin{array}{l}24.6- \\
48.0\end{array}$ & \\
\hline Mutant & 9 & 26.4 & $\begin{array}{l}15.2- \\
37.7\end{array}$ & & 29.1 & $\begin{array}{l}18.9- \\
39.2\end{array}$ & \\
\hline $\begin{array}{l}\text { EGFR mutation in } \\
\text { both primary lung } \\
\text { tumour and brain } \\
\text { metastatic tumour }\end{array}$ & & & & 0.159 & & & 0.380 \\
\hline Wild type & 16 & 16.5 & $\begin{array}{l}10.0- \\
23.0\end{array}$ & & 26.8 & $\begin{array}{l}18.4- \\
35.2\end{array}$ & \\
\hline Discordance & 3 & 37.2 & $\begin{array}{l}36.4- \\
38.1\end{array}$ & & 53.6 & $\begin{array}{l}53.6- \\
53.6\end{array}$ & \\
\hline Mutant type & 8 & 25.0 & $\begin{array}{l}12.9- \\
37.0\end{array}$ & & 27.5 & $\begin{array}{l}16.4- \\
38.6\end{array}$ & \\
\hline Size of BM & & & & 0.353 & & & 0.813 \\
\hline$\triangle 3 \mathrm{CM}$ & 13 & 22.4 & $\begin{array}{l}12.7- \\
32.0\end{array}$ & & 31.8 & $\begin{array}{l}22.8- \\
40.8\end{array}$ & \\
\hline$\geq 3 \mathrm{CM}$ & 14 & 23.1 & $\begin{array}{l}14.3- \\
31.8\end{array}$ & & 28.2 & $\begin{array}{l}16.9- \\
39.4\end{array}$ & \\
\hline $\begin{array}{l}\text { Intracranial } \\
\text { symptoms }\end{array}$ & & & & 0.633 & & & 0.698 \\
\hline Yes & 18 & 24.4 & $\begin{array}{l}15.5- \\
36.6\end{array}$ & & 36.3 & $\begin{array}{l}24.8- \\
47.7\end{array}$ & \\
\hline No & 9 & 21.7 & $\begin{array}{l}14.4- \\
29.0\end{array}$ & & 24.3 & $\begin{array}{l}16.2- \\
32.3\end{array}$ & \\
\hline Metastatic type & & & & 0.041 & & & 0.155 \\
\hline Synchronous ${ }^{3}$ & 15 & 17.5 & $\begin{array}{l}9.5- \\
25.6\end{array}$ & & 24.6 & $\begin{array}{l}16.4- \\
32.9\end{array}$ & \\
\hline Metachronous ${ }^{4}$ & 12 & 29.4 & $\begin{array}{l}21.6- \\
37.1\end{array}$ & & 44.6 & $\begin{array}{l}30.8^{-} \\
53.6\end{array}$ & \\
\hline
\end{tabular}

\footnotetext{
${ }^{1} \mathrm{ADC}$ :adenocarcinoma; ${ }^{2}$ Non -ADC: non-adenocarcinoma; ${ }^{3}$ Synchronous:brain metastases diagnosed with primary lung cancer; ${ }^{4}$ Metachronous:brain metastases diagnosed during the disease courses, ${ }^{5}$ EGFR-TKIs:first generation of tyrosine kinase inhibitors (TKIs): Gefitinib and Erlotinib; ${ }^{6}$ systemic chemotherapy plus local treatment.
} 


\begin{tabular}{|c|c|c|c|c|c|c|c|}
\hline Variable & $\mathbf{N}$ & PFS(months) & $95 \% \mathrm{Cl}$ & $P$ & OS(months) & $95 \% \mathrm{Cl}$ & $P$ \\
\hline Therapy modality & & & & 0.285 & & & 0.172 \\
\hline EGFR-TKIs ${ }^{5}$ & 7 & 26.2 & $\begin{array}{l}15.5- \\
36.9\end{array}$ & & 36.1 & $\begin{array}{l}27.3- \\
44.9\end{array}$ & \\
\hline $\begin{array}{l}\text { Conventional } \\
\text { therapy }{ }^{6}\end{array}$ & 20 & 22.8 & $\begin{array}{l}14.5- \\
31.0\end{array}$ & & 28.7 & $\begin{array}{l}18.0- \\
39.4\end{array}$ & \\
\hline \multicolumn{8}{|c|}{$\begin{array}{l}{ }^{1} \mathrm{ADC} \text { :adenocarcinoma; }{ }^{2} \text { Non -ADC: non-adenocarcinoma; }{ }^{3} \text { Synchronous:brain metastases } \\
\text { diagnosed with primary lung cancer; }{ }^{4} \text { Metachronous:brain metastases diagnosed during the disease } \\
\text { courses; }{ }^{5} \text { EGFR-TKIs:first generation of tyrosine kinase inhibitors (TKIs): Gefitinib and } \\
\text { Erlotinib; }{ }^{6} \text { systemic chemotherapy plus local treatment. }\end{array}$} \\
\hline
\end{tabular}

Heterogeneity of EGFR mutation in pulmonary tumour and paired brain metastatic tumour were found in our data,to our interesting, comparing to patients with consistent EGFR mutations,both mutant or wild, patients with inconsistent EGFR mutations have better outcomes,including PFS(37.2months vs 25.0 months vs 16.5 months, $P=0.159)$ and $O S(53.6$ months vs 27.5 months vs 26.8 months, $P=$ 0.380),which was showed in Table 5 and Fig. 4.Among the three discordant patients, two patients transfer from mutant to wild might had longer overall survival(53.6months,37.8months) than the patient transfer from negative to positive(16.7months)(Table 6).

Table 6

The characteristics for patients with inconsistent EGFR mutations.

\begin{tabular}{|c|c|c|c|c|c|c|c|}
\hline No & $\begin{array}{l}\text { EGFR mutation in } \\
\text { LC }\end{array}$ & $\begin{array}{l}\text { EGFR mutation in } \\
\text { BM }\end{array}$ & ${ }^{1} \mathrm{PFS} 1$ & ${ }^{2} \mathrm{PFS} 2$ & ${ }^{3} \mathrm{PFS}$ & ${ }^{4} \mathrm{OS}$ & Live status \\
\hline 1 & E746-A750 del & Wild & 36.6 & 53.6 & 36.6 & 53.6 & $\begin{array}{l}\text { Brain } \\
\text { progression }\end{array}$ \\
\hline 7 & L858R & Wild & 37.8 & 37.8 & 37.8 & 37.8 & Alive \\
\hline 10 & Wild & E746-A750 del & 16.7 & 14.3 & 14.3 & 16.7 & $\begin{array}{l}\text { Bone } \\
\text { progression }\end{array}$ \\
\hline \multicolumn{8}{|c|}{$\begin{array}{l}{ }^{1} \mathrm{PFS} 1 \text { :Time from surgery of brain metastatic tumour to intracranial tumour progression; }{ }^{2} \mathrm{PFS} 2: \text { Time } \\
\text { from surgery of brain metastatic tumour to extracranial tumour progression; }{ }^{3} \mathrm{PFS}: \text { Time from surgery } \\
\text { of brain metastatic tumour to any relapse; }{ }^{4} \mathrm{OS}: \text { Time from surgery of brain metastatic tumour to } \\
\text { cancer-related death. }\end{array}$} \\
\hline
\end{tabular}

All patients were divided into either the EGFR-TKIs treatment group or the conventional treatment group based on whether they received EGFR-TKIs. Compared to conventional therapy, EGFR-TKIs might have prolonged PFS of NSCLC patients with BMs (mPFS, 26.2 months vs 22.8 months, $P=0.285$ ). Similar results were observed for OS (mOS 36.1 months vs 28.7 months, $P=0.172$ )(Figure 5).

Response to EGFR-TKls according to EGFR mutation status. 
In this study, 7 patients received TKI treatment (Table 7). Five patients received TKIs as first-line treatment, and 2 patients received TKIs as second-line treatment. Of the 7 patients, EGFR mutations were detected simultaneously in 4 paired patients, and the mutational frequency was consistent. Among the patients who had EGFR mutations, TKI treatment provided a much longer PFS than that of EGFR wildtype patients (median 27.4 months vs 10.6 months), and the study found that TKIs also played a role in the extension of OS (27.4 months vs 20.8 months).

Table 7

Survival analysis in NSCLC patients with brain metastases who underwent EGFR-TKIs therapy.

\begin{tabular}{|c|c|c|c|c|c|c|c|}
\hline No & $\begin{array}{l}\text { EGFR mutation } \\
\text { in LC }\end{array}$ & $\begin{array}{l}\text { EGFR mutation in } \\
\text { BM }\end{array}$ & ${ }^{1}$ PFS1 & ${ }^{2} \mathrm{PFS} 2$ & ${ }^{3} \mathrm{PFS}$ & ${ }^{4} \mathrm{OS}$ & Status \\
\hline 9 & L858R & L858R & 36.0 & 36.0 & 36.0 & 36.0 & Alive \\
\hline 11 & E746-A750 del & E746-A750 del & 26.2 & 26.2 & 26.2 & 33.6 & Death \\
\hline 13 & Wild & Wild & 5.7 & 5.7 & 5.7 & 29.0 & Alive \\
\hline 14 & E746-A750 del & E746-A750 del & 39.7 & 39.7 & 39.7 & 43.0 & $\begin{array}{l}\text { Liver and brain } \\
\text { progression }\end{array}$ \\
\hline 16 & E746-A750 del & E746-A750 del & 9.3 & 9.3 & 9.3 & 9.3 & Death \\
\hline 19 & Wild & Wild & 30.6 & 30.0 & 30.0 & 30.6 & Lung progression \\
\hline 20 & Wild & Wild & 40.8 & 12.2 & 12.2 & 40.8 & Lung progression \\
\hline \multicolumn{8}{|c|}{$\begin{array}{l}{ }^{1} \mathrm{PFS} 1 \text { :Time from surgery of brain metastatic tumour to intracranial tumour progression; }{ }^{2} \mathrm{PFS} 2: \text { Time } \\
\text { from surgery of brain metastatic tumour to extracranial tumour progression; }{ }^{3} \mathrm{PFS} \text { :Time from surgery } \\
\text { of brain metastatic tumour to any relapse; }{ }^{4} \mathrm{OS} \text { :Time from surgery of brain metastatic tumour to } \\
\text { cancer-related death. }\end{array}$} \\
\hline
\end{tabular}

\section{Discussion}

A developing issue for targeted therapy in NSCLC is the identification of patients with EGFR mutations, who are the best candidates for clinical response[21]. However, only a small percentage of patients harboring EGFR mutations are sensitive to TKIs, suggesting that additional factors beyond EGFR mutation contribute to a patient's drug response. The heterogeneity of EGFR mutations has been identified as a potential cause of failure in targeted therapy, although the existence of such heterogeneity remains controversial[21]. Therefore, it is of clinical significance to elucidate the EGFR mutational heterogeneity between primary tumour and metastatic tumour.

The heterogeneity of EGFR mutation status has long been assumed in NSCLC. However, few studies focus on EGFR mutation between brain metastases and primary lung tumours. A previous study including 25 patients of Asian ethnicity showed a discordance of $32 \%$ between the primary tumour and corresponding brain metastatic tumour[17], whereas another two studies showed different results[16, 18]. Another related research was designed in Chinese patients, in this study, about $80 \%$ concordance were found in 
mutations of major driver gene between primary lung tumour and paired BM,they reported the significant correlation between PI3K signaling and increased BM risk in NSCLC patients[22]. Our study suggested that heterogeneity of EGFR mutation status occurred in primary tissue and paired brain metastatic tumour, and the discordance of EGFR mutation status was $11.1 \%(3 / 27)$. It seems that EGFR mutational heterogeneity exists in NSCLC with BM.

The other study came to the opposite conclusion, and their results proved that EGFR mutational heterogeneity was rare in lung adenocarcinoma. Yatabe et al[23] reported that the heterogeneous distribution of EGFR mutations in lung adenocarcinoma was rare. They believed that phenomenon of heterogeneity resulted in intratumoural distribution. In the present study,heterogeneity was observed in three patients, two of the cases were adenocarcinoma.

The EGFR mutation was evaluated again from different parts of the tumour to reduce the influence of intratumoural mutational heterogeneity, and similar results were obtained again by the second evaluation. The EGFR mutation status might change during disease progression, some study proved that the EGFR -mutant patients were more likely to experience brain metastases than wild ones[24, 25]. Another Meta analysis study reported that in metachrounous brain metastasis patients, compared with EGFR wild type patients, EGFR mutant patients possessed a significantly higher risk of $B M(O R=$ $2.01 ; 95 \% \mathrm{Cl}, 1.56-2.59 ; \mathrm{P} \leq 0.001)$,however,just a trend of increasing risk were observed in synchronous brain metastasis without significant difference $(\mathrm{OR}=1.38 ; 95 \% \mathrm{Cl}, 0.98-1.94 ; \mathrm{P} \leq 0.066)$. Moreover,EGFR mutant patients had longer overall survival than wild type patients in initial BM,but no significant difference in patients with subsequent BMs[19]. All the above patients' EGFR mutation status were detected in the primary tumour,it seemed that EGFR involved in the process of brain metastasis and also affected the prognosis of patients.

Luo et.al [26] reported that EGFR mutation status in brain metastatic tumour had no influence on PFS and OS of 136 Chinese patients with BM,although there was no significant difference,median PFS and OS of wild type patients were longer than mutant patients(PFS:12.0months vs $10.0 \mathrm{months}$; OS:24.5months vs 15.0mongths). In our study,patients harbored EGFR mutant in primary lung tumour had longer PFS (mPFS, 27.8 months vs 17.4 months, $P=0.156$ ) and OS (mPFS,34.9months vs 27.6 months, $P=0.606$ ), but for matched brain metastatic tumour, patients with negative mutations had a longer OS of 34.9 months compared with 27.6 months in EGFR positive patients $(P=0.944)$.Our research and the related study indicated that EGFR negative in brain metastatic tumour yielded more overall survival of NSCLC patients with BM.

Our team previously reported a retrospective study of 282 NSCLC patients with BM, and EGFR mutations were detected in 109 tissue samples. The frequency of EGFR mutation was 50\% (55/109). Survival analysis showed that patients with TKIs treatment had longer intracranial progression-free survival (19.8 months versus 12.0 months, $\mathrm{P} \otimes 0.0001$ ) and extracranial progression-free survival (19.6 months versus 12.3 months, $P \otimes 0.0001)$, compared to those of patients who underwent conventional treatment. Further analysis found that in the TKI group, patients with EGFR mutations had better extracranial disease 
control (median, 20.4 months vs 14.1 months, $P=0.032$ )[20]. Although only 7 patients who received TKIs were enrolled in this study, we still proved that TKls therapy could improve the survival of NSCLC with BM compared to that of conventional therapy, especially for patients harboring mutant EGFR.

This study had the following limitations. First, the sample size was too small, and only 27 patients were enrolled in this study due to the limited number of brain metastatic tumour samples obtained. Second, subject to technical standards at past, AMRS technology was used to detect EGFR mutations in primary tumours and corresponding metastatic tumours, and we know that the detection sensitivity was different between the methods. The last but most important limitation was that the tissues that presented heterogeneity were evaluated twice to reduce the effect of intratumoural mutational heterogeneity, but this was still not enough sampling.

The heterogeneity of EGFR mutations between primary tumour and brain metastatic tumour was observed in our study, especially for metachronous brain tumour. Two patients "lose" the EGFR mutation during the barin metastases,but one patient "gain" the EGFR mutation in this process. Patients harbored wild type EGFR mutation in brain metastasis might have better outcomes,especially for patients whose positive mutation status transferred to wild.

\section{Abbreviations}

EGFR

Epidermal growth factor receptor; BM:brain metastasis; NSCLC:Non-small cell lung cancer; ARMS:amplification refraction mutation system; WBRT:Whole brain radiation therapy; SRS:stereotactic radiosurgery; PFS:Progression free survival; OS:Overall survival; TKIs:Tyrosine kinase inhibitors; BBB:blood-brain barrier.

\section{Declarations}

\section{Acknowledgements}

Not applicable.

\section{Funding}

This study was supported by Wu Jieping Medical Foundation (320.6750.17527).

\section{Availability of data and materials}

All data are fully available without restriction.

\section{Authors'contributions}

1. Conception and design: Tao Jiang,Lanjun Zhang; 
2. Provision of study materials or patients: Tao Jiang, Lanjun Zhang;

3. Collection and assembly of data: Shuonan Xu, Jianfei Zhu, Daixing Zhong, Yanmin Xia, Yingsheng Wen;

4. Data analysis and interpretation: Tao Jiang,Lanjun Zhang;

5. Manuscript writing: Shuonan Xu, Jianfei Zhu, Daixing Zhong;

(VI) Final approval of manuscript: All authors.

\section{Ethics approval and consent to participate}

This study was approved by Ethics Committee of the Second Affiliated Hospital of Air Force Medical University. Patient data were de-identified and anonymized before analysis.

\section{Consent for publication}

Informed consent for publication was obtained in all cases (patients or their relative, if the patients have died). They all understand that this journal is freely available on the web and under the license which the journal uses material published can be redistributed freely and used for any legal purpose.

\section{Competing interests}

We declare that we have no conflicts of interest.

\section{References}

1. Wang $H$, Wang Z, Zhang G, Zhang M, Zhang X, Li H, Zheng X, Ma Z. Driver genes as predictive indicators of brain metastasis in patients with advanced NSCLC: EGFR, ALK, and RET gene mutations. Cancer Med. 2020;9:487-95.

2. Andrews DW, Scott CB, Sperduto PW, Flanders AE, Gaspar LE, Schell MC, Werner-Wasik M, Demas W, Ryu J, Bahary JP, et al. Whole brain radiation therapy with or without stereotactic radiosurgery boost for patients with one to three brain metastases: phase III results of the RTOG 9508 randomised trial. Lancet. 2004;363:1665-72.

3. Gui Q, Liu J, Li D, Xu C. Prolonged survival of patients with EGFR-mutated non-small cell lung cancer with solitary brain metastases treated with surgical resection of brain and lung lesions followed by EGFR TKIs. World J Surg Oncol. 2017;15:184.

4. Eichler AF, Chung E, Kodack DP, Loeffler JS, Fukumura D, Jain RK. The biology of brain metastasestranslation to new therapies. Nat Rev Clin Oncol. 2011;8:344-56.

5. McKillop D, Partridge EA, Hutchison M, Rhead SA, Parry AC, Bardsley J, Woodman HM, Swaisland HC. Pharmacokinetics of gefitinib, an epidermal growth factor receptor tyrosine kinase inhibitor, in rat and dog. Xenobiotica. 2004;34:901-15. 
6. Meany HJ, Fox E, McCully C, Tucker C, Balis FM. The plasma and cerebrospinal fluid pharmacokinetics of erlotinib and its active metabolite (OSI-420) after intravenous administration of erlotinib in non-human primates. Cancer Chemother Pharmacol. 2008;62:387-92.

7. Nishino M, Soejima K, Mitsudomi T. Brain metastases in oncogene-driven non-small cell lung cancer. Transl Lung Cancer Res. 2019;8:298-307.

8. Ramalingam SS, Vansteenkiste J, Planchard D, Cho BC, Gray JE, Ohe Y, Zhou C, Reungwetwattana T, Cheng Y, Chewaskulyong B, et al. Overall Survival with Osimertinib in Untreated, EGFR-Mutated Advanced NSCLC. N Engl J Med. 2020;382:41-50.

9. Pao W, Miller VA, Politi KA, Riely GJ, Somwar R, Zakowski MF, Kris MG, Varmus H. Acquired resistance of lung adenocarcinomas to gefitinib or erlotinib is associated with a second mutation in the EGFR kinase domain. Plos Med. 2005;2:e73.

10. Janne PA. Challenges of detecting EGFR T790M in gefitinib/erlotinib-resistant tumours. Lung Cancer. 2008;60(Suppl 2):3-9.

11. Suda K, Murakami I, Katayama T, Tomizawa K, Osada H, Sekido Y, Maehara Y, Yatabe Y, Mitsudomi T. Reciprocal and complementary role of MET amplification and EGFR T790M mutation in acquired resistance to kinase inhibitors in lung cancer. Clin Cancer Res. 2010;16:5489-98.

12. Taniguchi K, Uchida J, Nishino K, Kumagai T, Okuyama T, Okami J, Higashiyama M, Kodama K, Imamura F, Kato K. Quantitative detection of EGFR mutations in circulating tumor DNA derived from lung adenocarcinomas. Clin Cancer Res. 2011;17:7808-15.

13. Nakano H, Soda H, Takasu M, Tomonaga N, Yamaguchi H, Nakatomi K, Fujino S, Hayashi T, Nakamura Y, Tsukamoto K, Kohno S. Heterogeneity of epidermal growth factor receptor mutations within a mixed adenocarcinoma lung nodule. Lung Cancer. 2008;60:136-40.

14. Schmid K, OehI N, Wrba F, Pirker R, Pirker C, Filipits M. EGFR/KRAS/BRAF mutations in primary lung adenocarcinomas and corresponding locoregional lymph node metastases. Clin Cancer Res. 2009;15:4554-60.

15. Matsumoto S, Takahashi K, Iwakawa R, Matsuno Y, Nakanishi Y, Kohno T, Shimizu E, Yokota J. Frequent EGFR mutations in brain metastases of lung adenocarcinoma. Int $\mathrm{J}$ Cancer. 2006;119:1491-4.

16. Daniele L, Cassoni P, Bacillo E, Cappia S, Righi L, Volante M, Tondat F, Inghirami G, Sapino A, Scagliotti GV, et al. Epidermal growth factor receptor gene in primary tumor and metastatic sites from non-small cell lung cancer. J Thorac Oncol. 2009;4:684-8.

17. Gow CH, Chang YL, Hsu YC, Tsai MF, Wu CT, Yu CJ, Yang CH, Lee YC, Yang PC, Shih JY. Comparison of epidermal growth factor receptor mutations between primary and corresponding metastatic tumors in tyrosine kinase inhibitor-naive non-small-cell lung cancer. Ann Oncol. 2009;20:696-702.

18. Matsumoto S, Takahashi K, Iwakawa R, Matsuno Y, Nakanishi Y, Kohno T, Shimizu E, Yokota J. Frequent EGFR mutations in brain metastases of lung adenocarcinoma. Int $\mathrm{J}$ Cancer. 2006;119:1491-4. 
19. Li L, Luo S, Lin H, Yang H, Chen H, Liao Z, Lin W, Zheng W, Xie X. Correlation between EGFR mutation status and the incidence of brain metastases in patients with non-small cell lung cancer. $J$ Thorac Dis. 2017;9:2510-20.

20. Cai L, Zhu JF, Zhang XW, Lin SX, Su XD, Lin P, Chen K, Zhang LJ. A comparative analysis of EGFR mutation status in association with the efficacy of TKI in combination with WBRT/SRS/surgery plus chemotherapy in brain metastasis from non-small cell lung cancer. J Neurooncol. 2014;120:423-30.

21. Mok TS, Wu YL, Thongprasert S, Yang CH, Chu DT, Saijo N, Sunpaweravong P, Han B, Margono B, Ichinose $Y$, et al. Gefitinib or carboplatin-paclitaxel in pulmonary adenocarcinoma. N Engl J Med. 2009;361:947-57.

22. Wang H, Ou Q, Li D, Qin T, Bao H, Hou X, Wang K, Wang F, Deng Q, Liang J, et al. Genes associated with increased brain metastasis risk in non-small cell lung cancer: Comprehensive genomic profiling of 61 resected brain metastases versus primary non-small cell lung cancer (Guangdong Association Study of Thoracic Oncology 1036). Cancer-Am Cancer Soc. 2019;125:3535-44.

23. Yatabe Y, Matsuo K, Mitsudomi T. Heterogeneous distribution of EGFR mutations is extremely rare in lung adenocarcinoma. J Clin Oncol. 2011;29:2972-7.

24. Hsu F, De Caluwe A, Anderson D, Nichol A, Toriumi T, Ho C. EGFR mutation status on brain metastases from non-small cell lung cancer. Lung Cancer. 2016;96:101-7.

25. Baek MY, Ahn HK, Park KR, Park HS, Kang SM, Park I, Kim YS, Hong J, Sym SJ, Park J, et al. Epidermal growth factor receptor mutation and pattern of brain metastasis in patients with nonsmall cell lung cancer. Korean J Intern Med. 2018;33:168-75.

26. Luo D, Ye X, Hu Z, Peng K, Song Y, Yin X, Zhu G, Ji Q, Peng Y. EGFR mutation status and its impact on survival of Chinese non-small cell lung cancer patients with brain metastases. Tumour Biol. 2014;35:2437-44.

\section{Figures}




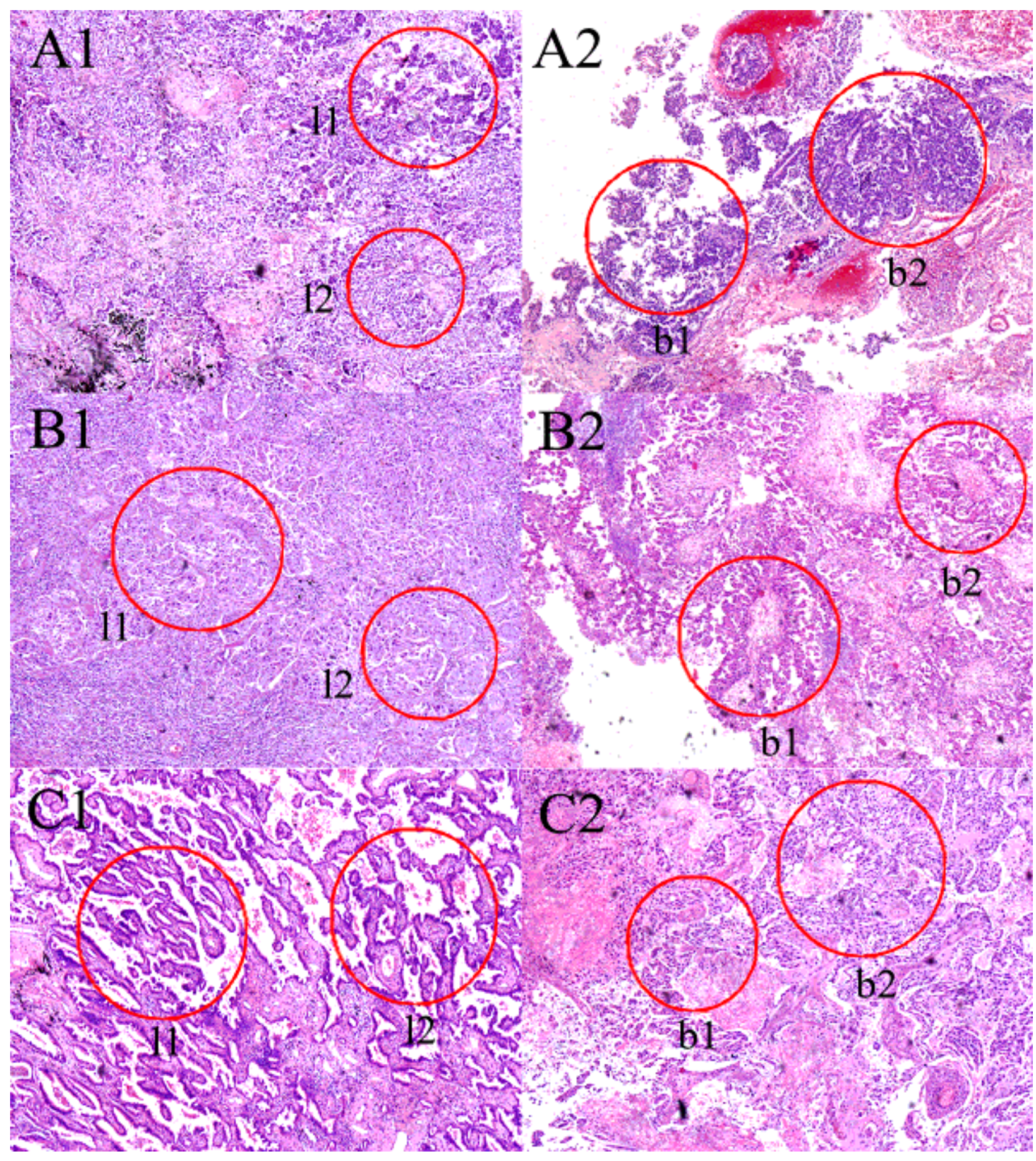

Figure 1

The different sites of lung primary tumor and paired brain metastatic tumor in the three with inconsistent mutations. 

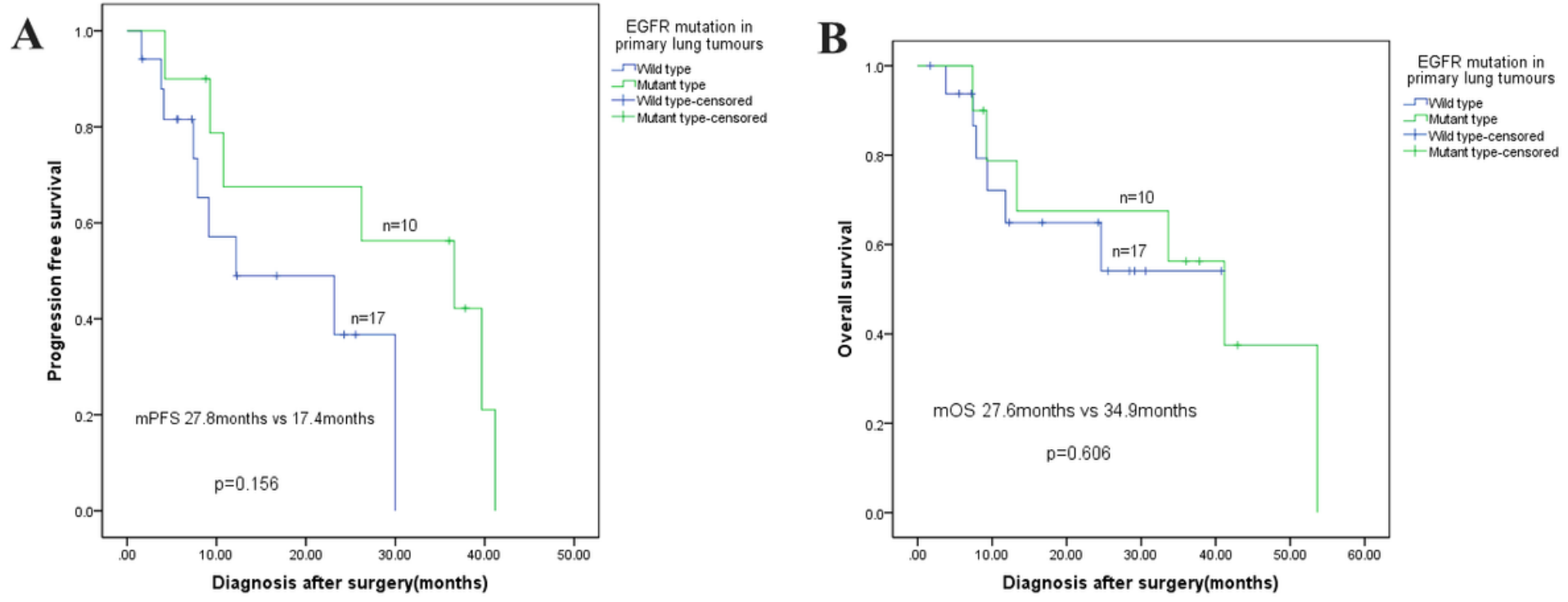

Figure 2

PFS and OS curve of NSCLC with BMs according to mutations status in lung primary tumour.
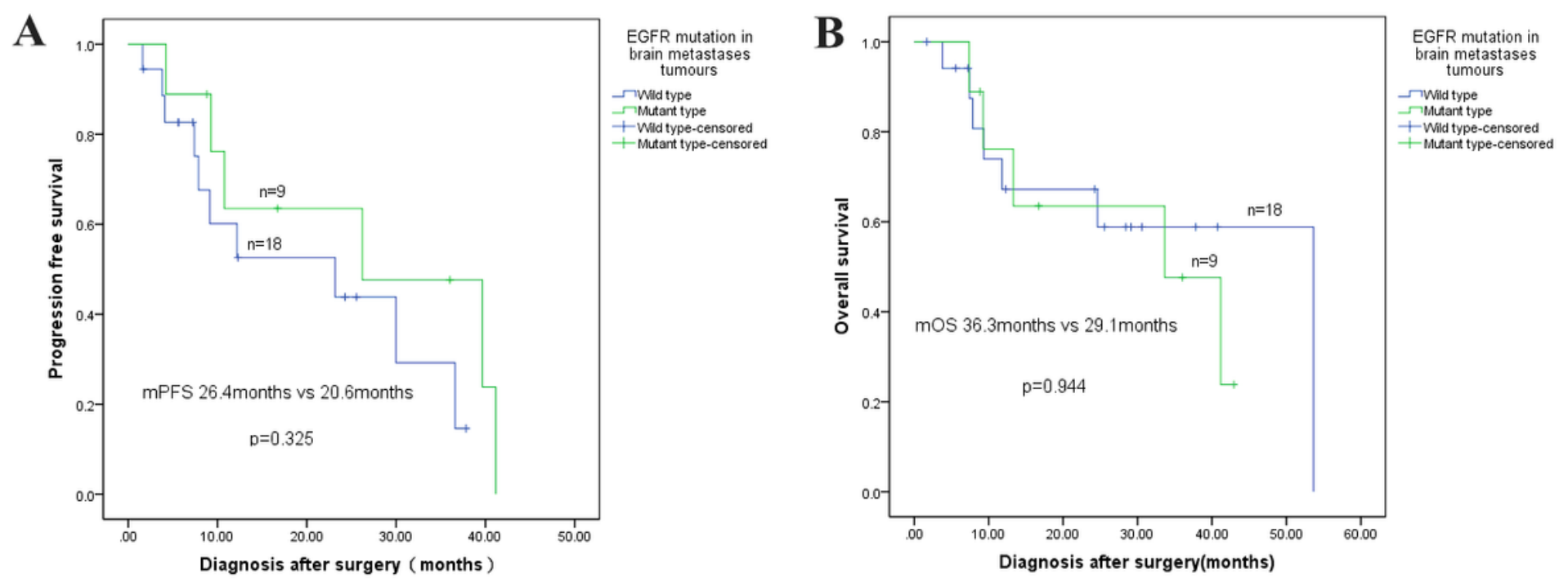

Figure 3

PFS and OS curve of NSCLC with BMs according to mutations status in brain metastatic tumour. 

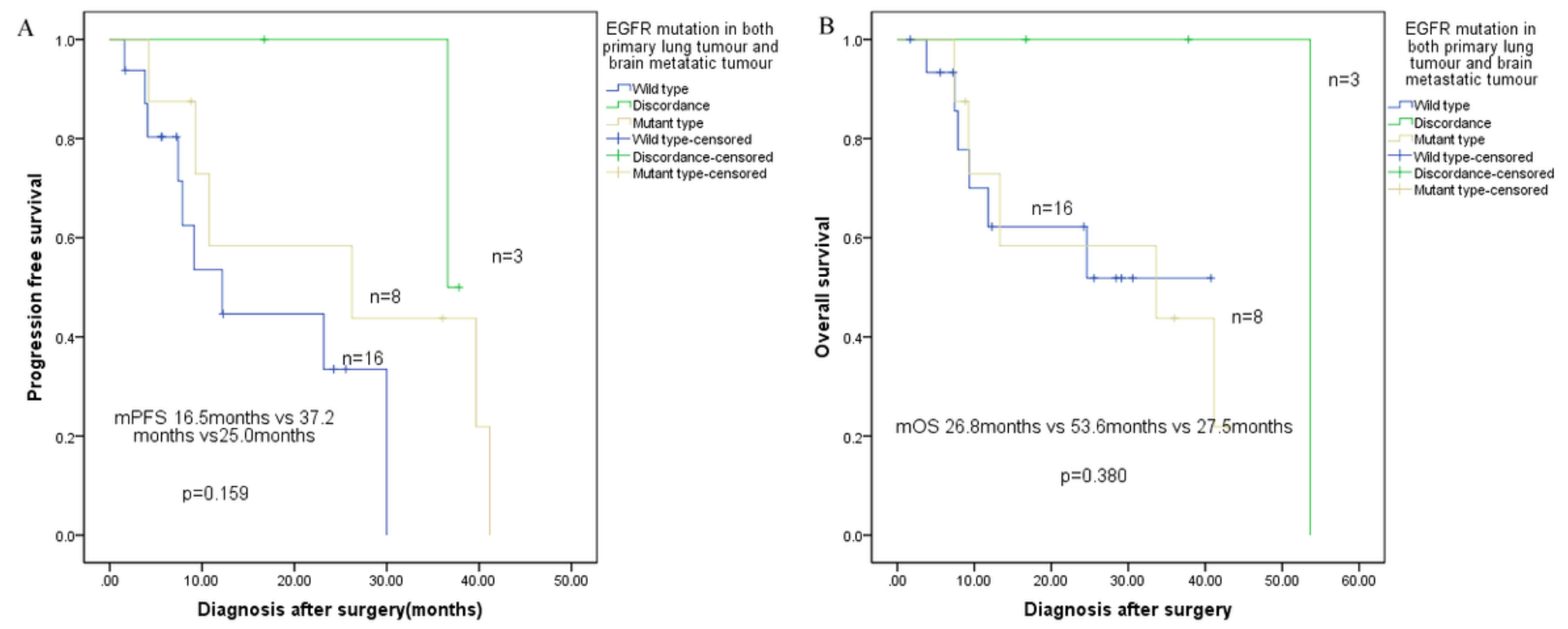

Figure 4

PFS and OS curve of NSCLC with BMs according to the consistence of mutations status in lung primary tumour and paired brain metastatic tumour.
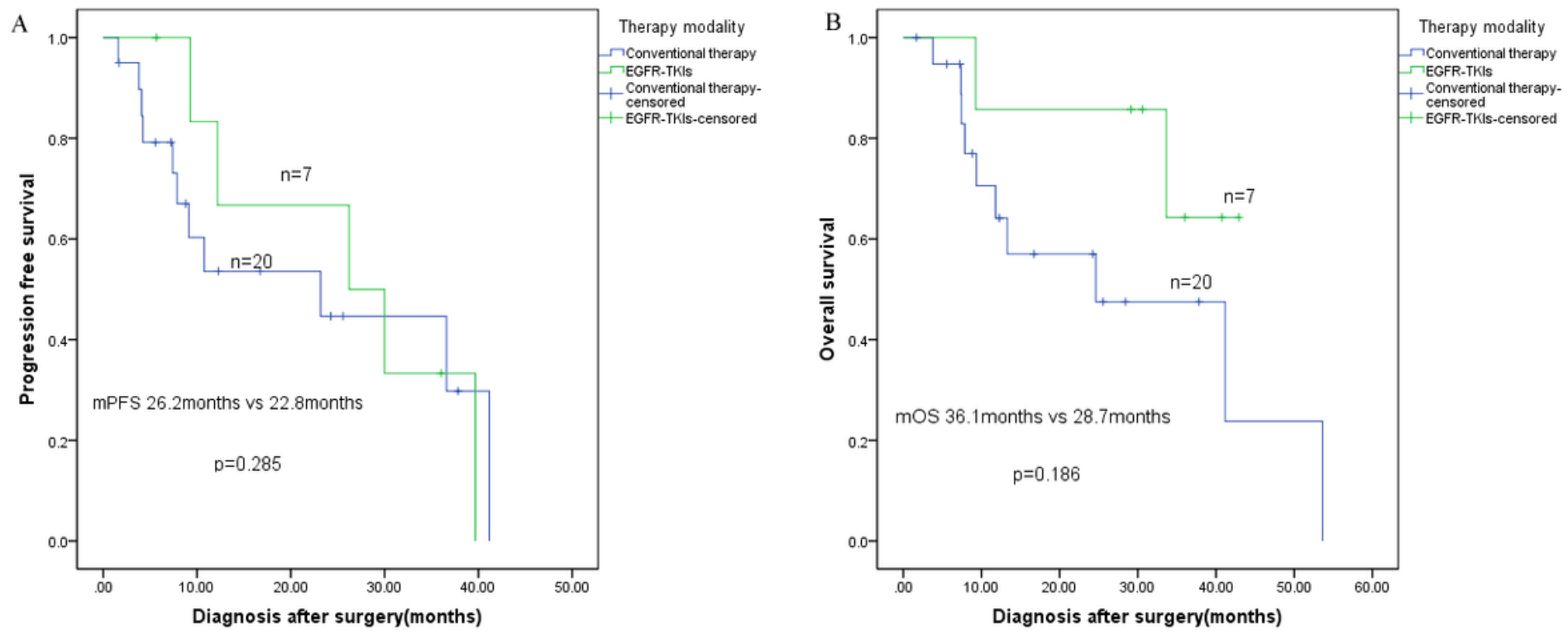

Figure 5

PFS and OS curve of NSCLC with BMs according to the therapeutic modality. 